\title{
Surface Assisted Laser Desorption/Ionization on Two-Layered Amorphous Silicon Coated Hybrid Nanostructures
}

\author{
Ville Jokinen, ${ }^{\text {a,b }}$ Susanna Aura, ${ }^{\mathrm{b}}$ Laura Luosujärvi, ${ }^{\mathrm{c}}$ Lauri Sainiemi, ${ }^{\mathrm{b}}$ \\ Tapio Kotiaho, ${ }^{\text {c,d }}$ Sami Franssila, ${ }^{b}$ and Marc Baumann ${ }^{a}$ \\ ${ }^{a}$ Protein Chemistry Unit, Institute of Biomedicine, University of Helsinki, Helsinki, Finland \\ ${ }^{\mathrm{b}}$ Department of Micro- and Nanosciences, Helsinki University of Technology, Espoo, Finland \\ ${ }^{\mathrm{c}}$ Laboratory of Analytical Chemistry, Department of Chemistry, University of Helsinki, Helsinki, Finland \\ ${ }^{\mathrm{d}}$ Faculty of Pharmacy, Division of Pharmaceutical Chemistry, University of Helsinki, Helsinki, Finland
}

Matrix-free laser desorption/ionization was studied on two-layered sample plates consisting of a substrate and a thin film coating. The effect of the substrate material was studied by depositing thin films of amorphous silicon on top of silicon, silica, polymeric photoresist SU-8, and an inorganic-organic hybrid. Des-arg'-bradykinin signal intensity was used to evaluate the sample plates. Silica and hybrid substrates were found to give superior signals compared with silicon and SU-8 because of thermal insulation and compatibility with amorphous silicon deposition process. The effect of surface topography was studied by growing amorphous silicon on hybrid micro- and nanostructures, as well as planar hybrid. Compared with planar sample plates, micro- and nanostructures gave weaker and stronger signals, respectively. Different coating materials were tested by growing different thin film coatings on the same substrate. Good signals were obtained from titania and amorphous silicon coated sample plates, but not from alumina coated, silicon nitride coated, or uncoated sample plates. Overall, the strongest signals were obtained from oxygen plasma treated and amorphous silicon coated inorganic-organic hybrid, which was tested for peptide-, protein-, and drug molecule analysis. Peptides and drugs were analyzed with little interference at low masses, subfemtomole detection levels were achieved for des-arg'-bradykinin, and the sample plates were also suitable for ionization of small proteins. (J Am Soc Mass Spectrom 2009, 20, 1723-1730) @ 2009 American Society for Mass Spectrometry

S urface-assisted laser desorption/ionization (SALDI) is an ionization technique for mass spectrometry (MS) where an analyte, deposited on a surface, is ionized upon exposure to laser light without cocrystallization with matrix molecules. The terminology of the field is somewhat unclear, but we take the term SALDI to refer to both particle suspension matrices as well as desorption ionization on porous silicon [1] (DIOS), and similar monolithic "matrixfree" approaches. SALDI offers several advantages compared with the ubiquitous matrix-assisted laser desorption/ionization (MALDI) [2], such as greatly reduced or eliminated background signals at low molecular masses [1], less pronounced "sweet-spot" effect, and a simpler analysis procedure, which allows easier integration with other functionalities, e.g., in situ chromatographic separations [3] and analyte capture and purification [4]. The detection limits of SALDI techniques for small peptides are often comparable to MALDI (high attomoles), and in some cases can even greatly surpass

Address reprint requests to Mr. V. Jokinen, Protein Chemistry Unit, Institute of Biomedicine, Haartmaninkatu 8, P.O. Box 63, 00014 University of Helsinki, Finland. E-mail: ville.p.jokinen@helsinki.fi them [4]. The feasibility of SALDI-MS has been demonstrated in the analysis of many different types of analytes, including peptides [1], trypsin digests $[5,6]$, proteins up to $24 \mathrm{kDa}$ in mass [7, 8], small drug molecules [4, 9], and polymers [6].

Early studies related to SALDI focused on the use of inorganic matrices, such as cobalt nanoparticles suspended in glycerol by Tanaka et al. [10] and graphite microparticles also suspended in glycerol by Sunner et al. [11]. However, with the success of DIOS, where analytes are ionized on a monolithic porous silicon surface, the trend has been toward investigating matrixfree methods, where the surfaces themselves, in addition to the possible contribution of the solvent and contaminants, contain the properties necessary for efficient desorption and ionization of the analytes.

Since then, a multitude of different surfaces have been reported to work in SALDI. Silicon has remained the most common material and, in addition to porous silicon, SALDI has been reported on silicon nanowires [12], nanopillars [9], nanocavity arrays [13], nanogroove arrays [14], column/void silicon thin films [15], and amorphous silicon $(\alpha-\mathrm{Si})$ [16]. However, silicon as a material is not a necessary requirement SALDI, which 
has also been reported on etched carbon [17], germanium nanodots [6], titania nanotube arrays [18], and nanocrystalline titania [7], carbon nanotube arrays [19], metal coated porous alumina [8], and cobalt and graphite particle suspensions $[10,11]$.

The desorption and ionization mechanisms in SALDI remain mostly unknown, but multiple studies have identified factors that are conducive to efficient ion generation. Good absorptivity at the wavelength of the laser is widely accepted as a necessity for transferring energy from the laser to the substrate. Low thermal conductivity helps confine the heat [8] near the surface, but high thermal conductivity at the surface has been claimed to be conducive for transferring energy from the surface to the analytes [20]. The absorbed laser light then heats the surface, which has been identified to play a key role in the desorption process [17, 21, 22]. Thermal evaporation of the analyte and residual solvents has been suggested to be the desorption mechanism on porous silicon [21], but recently the role of thermally activated morphologic transitions have been emphasized by Northen et al. [22], who note that the ionization threshold typically does not depend on the properties of the analyte, as would be expected for a strictly thermal desorption process. In addition to thermally driven desorption mechanisms, field desorption driven by the laser light, possibly amplified by surface nanostructures, has been proposed $[6,12,17]$. The origin of the protons (for protonated analytes) has been suggested to be either the surface itself [16, 22], residual solvents, acids or adsorbed hydrocarbons [1, 21], or both the surface and the solvent [23]. The role of surface topography in the desorption-ionization process remains unclear. Nanopores have been claimed [21] to be fundamental to efficient SALDI through elevating the background pressure and lowering the dimensionality of the desorption plume, leading to overall higher plume densities. On the other hand, it has also been argued that the role of pores is limited to storing and resupplying the surface with analyte molecules [17], while it is actually a disordered near- surface layer with a high density of dangling bonds that is essential for efficient SALDI, as demonstrated by nonporous amorphous silicon [16]. In addition to nanopores, submicrometer surface roughness of any kind seems to correlate with efficient SALDI [14, 17].

Our approach to studying the SALDI mechanism and designing efficient SALDI sample plates is fabricating and performing SALDI-MS measurements on sample plates consisting of two layers: a substrate (thickness $>1 \mu \mathrm{m}$ ) and a thin film coating (thickness $<100$ $\mathrm{nm})$. In this way, each phenomenon for which the characteristic length scale is smaller than the thickness of the coating will be determined by the properties of the coating layer, and vice versa. The big advantage of this approach is that the properties of the two layers can be varied independent of each other, which is helpful both for mechanistic studies and to experimental optimization of the SALDI sample plates. A similar ap- proach has been reported by Wada et al. [8], who studied various metal coatings on top of porous alumina. In this study, we varied the material properties of the substrate layer and the coating layer as well as the topography of the sample plates. The substrate layer materials that were tested were crystalline silicon, amorphous silicon dioxide, an organic polymer SU-8, and an inorganic-organic hybrid material, while the coatings were amorphous silicon $(\alpha-\mathrm{Si})$, silicon nitride, titanium dioxide, and aluminum oxide. Substrate topography was tested by growing the coatings on top of micro- and nanostructured substrates in addition to planar surfaces. The optimized surfaces were tested for SALDI-MS of peptides, small proteins, and drug molecules.

\section{Experimental}

\section{Materials}

Angiotensin I, angiotensin II, bombesin, Substance P, and des-arg ${ }^{9}$-bradykinin were from PeptaNova (Sandhausen, Germany), cytochrome $c$ and $\beta$-lactoglobulin were from Sigma-Aldrich Chemie GmbH (Munich, Germany) and verapamil hydrochloride (98\% purity) was from Aldrich Chem. Co (Milwaukee, WI, USA). Acetonitrile (ACN) and HPLC grade methanol were from Rathburn Chemicals Ltd. (Walkerburn, Scotland), Citric acid monohydrate was from Merck (Darmstadt, Germany) and $\alpha$-cyano-4-hydroxy-cinnamic acid (CHCA) was from Sigma (St. Louis, MO, USA). Water was purified by ELGA USF Maxima water purifier. SU-8 50 and hybrid material Ormocomp US S4 were from Micro resist technology (Berlin, Germany), and silicon wafers $(10 \mathrm{~cm}$ SSP) were from University Wafer (Boston, MA).

\section{Substrate Fabrication}

All fabrication was carried out in the clean room under class 10-100 conditions. Before any fabrication, the wafers were cleaned with RCA cleaning. Silicon dioxide substrate was fabricated by thermal oxidation of a silicon wafer. SU-8 substrate was fabricated on top of a silicon wafer by standard fabrication process for a 10 $\mu \mathrm{m}$ layer and hardbaked on a hotplate at $200{ }^{\circ} \mathrm{C}$ for $1 \mathrm{~h}$. Planar hybrid substrate (15 $\mu \mathrm{m}$ thick) was fabricated on top of a silicon wafer by a standard process for Ormocomp [24]. The Ormocomp hybrid is a versatile material that consists of an inorganic silica backbone crosslinked by organic domains, and the sol-gel technology used to create the material also allows the incorporation of functional organic groups and metals [25]. The hybrid micro- and nanopillars [26] were fabricated by UVembossing, and the micropillars were $5 \mu \mathrm{m}$ high and 2 $\mu \mathrm{m}$ in diameter cylinders in a $2 \mu \mathrm{m}$ spacing regular lattice, and the nanopillars were cone shaped, about 500 $\mathrm{nm}$ wide at the base and $1 \mu \mathrm{m}$ high in an irregular lattice. The oxygen plasma treatment for the hybrid was made in a reactive ion etch (RIE) reactor (Plasmalab 80+, Oxford Instruments Plasma Technology, Oxford, 
Abingdon, UK) at $200 \mathrm{~W}, 45 \mathrm{sccm} \mathrm{O}_{2}, 5 \mathrm{sccm}$ argon, and $10 \mathrm{~min}$ processing time for planar hydrid and $1 \mathrm{~min}$ processing time for hybrid micropillars and nanopillars.

\section{Coatings and Surface Characterization}

Amorphous silicon and silicon nitride were grown in a PECVD reactor (Plasmalab 80+). The deposition parameters for amorphous silicon were: temperature $200{ }^{\circ} \mathrm{C}$, power $5 \mathrm{~W}$, pressure $2000 \mathrm{mTorr}, 14 \mathrm{sccm} \mathrm{SiH}_{4}$, and 266 sccm He. The deposition rate was determined by profilometry and reflectometry to be $\approx 8 \mathrm{~nm} / \mathrm{min}$. Aluminum oxide and titanium oxide were deposited by atomic layer deposition (ALD) (TFS 500; Beneq Oy, Vantaa, Finland). Scanning electron microscopy (SEM) (Supra 40, Carl Zeiss SMT, Oberkochen, Germany) and atomic force microscopy (AFM) (NTEGRA; NT-MDT Co., Moscow, Russia) were used to characterize the surfaces.

\section{Storage of Sample Plates}

The sample plates were stored in air inside the clean room before MS measurements. Sample plates were used for a maximum of $1 \mathrm{wk}$ after being brought out of the clean room and, with the exception of testing the effect of storage, were used for a maximum of 2 mo after the final coating was done. Before each measurement, the sample plates were cleaned by rinsing with methanol.

\section{MS Measurements}

Peptides and proteins were stored as $200 \mu \mathrm{M}$ solutions in water and stored at $-20^{\circ} \mathrm{C}$. Analytes were diluted in purified water, and the concentrations of analytes used are mentioned in the text. The citric acid additive was dissolved in water to $1 \mathrm{mM}$ concentration and prespotted on the sample plates before analyte deposition. Analyte solutions were pipetted directly on the sample plates in $1 \mu \mathrm{L}$ aliquots. No special liquid confinement was used and the sample spot size was usually about 2 $\mathrm{mm}$ in diameter on amorphous silicon coated sample plates. The sample plates were mounted on a standard steel MALDI plate with double sided conductive tape. Bruker Daltonics (Bremen, Germany) Autoflex III MALDI-TOF mass spectrometer with SmartBeam laser ( $\lambda=355 \mathrm{~nm}, 7 \mathrm{~ns}$ pulse duration, $50 \mathrm{~Hz}$ repetition rate) was in all measurements. For peptides and drugs, positive reflectron mode was used, and the detected mass range was either 800 Da-2400 Da with suppression below $500 \mathrm{Da}$, or 40-2000 Da without suppression. Unless the spectra specifically show the low mass range, the former was used. For proteins, positive linear mode was used. A mass spectrum was collected by summing $10 \times 50$ laser shots from each sample spot, except in the substrate material test, where $10 \times 20$ laser shots were summed. Blank samples were measured in the SALDI sensitivity analysis experiments. For MALDI analysis the matrix was CHCA dissolved in $40 \%$ ACN with $0.1 \%$ TFA, and $5 \times 50$ laser shots were summed in positive reflectron mode.

\section{Evaluation of Sample Plates}

Signal intensity of the $[\mathrm{M}+\mathrm{H}]^{+}$peak of des-arg ${ }^{9}-$ bradykinin was used to evaluate the ionization performance of the various surfaces. The analyte volume and concentration was kept the same for all surfaces, but the laser fluence was optimized for each surface separately in the substrate material experiment. This laser fluence optimization was done based on obtaining the strongest possible $[\mathrm{M}+\mathrm{H}]^{+}$peak of des-arg' ${ }^{9}$-bradykinin. The signal intensity was reasonably uniform within the sample deposition spot with most of the spot producing signals between $80 \%-100 \%$ of the maximum intensity of that spot (Figure S1, Supplementary Material, which can be found in the electronic version of this article), and signal intensity variation was further reduced by manually collecting a sum spectra from 10 different places within the spot. Spot to spot variation was reduced by always measuring from multiple sample spots on the same sample plates, and averaging the results. The substrate material test, substrate topography test, coating material test, and the des-arg'-bradykinin sensitivity test were also repeated on at least two different batches of chips to control for random variations in the fabrication process and to verify the results.

\section{Results and Discussion}

\section{Substrate Material}

Four different substrates were coated with a thin $\alpha$-Si layer for testing the effect of the material properties of the substrate. The substrate materials in this experiment were crystalline silicon, amorphous thermal silicon dioxide, polymeric photoresist SU-8, and an organic-inorganic hybrid. The substrates were coated simultaneously in the PECVD chamber to ensure as homogeneous as possible deposition conditions for the coating. We found that on these essentially planar sample plates, the analyte signal persisted for $\approx 50$ laser shots. Typical spectra on each substrate are shown in Figure 1, and Table 1 summarizes the average signal intensities of the protonated molecular ion peak of des-arg'-bradykinin and the root-mean-square (rms) roughness of the $40 \mathrm{~nm}$ thick $\alpha$-Si layer, as measured by AFM.

As reported by Alimpiev et al. [16], amorphous silicon is clearly SALDI active since none of the four substrates gave analyte signals without the $\alpha$-Si coating. On silicon, $\mathrm{SiO}_{2}$, and hybrid material, the analyte was detected as a single protonated species, while on the SU-8 substrate, the most intensive peak was the potassium adduct peak followed by the sodium adduct peak and the protonated molecular ion peak. For this reason, SU-8 was excluded from the quantitative analysis in Table 1. It is not known why SU-8 behaved differently compared with the other materials. As neither SU-8 nor 


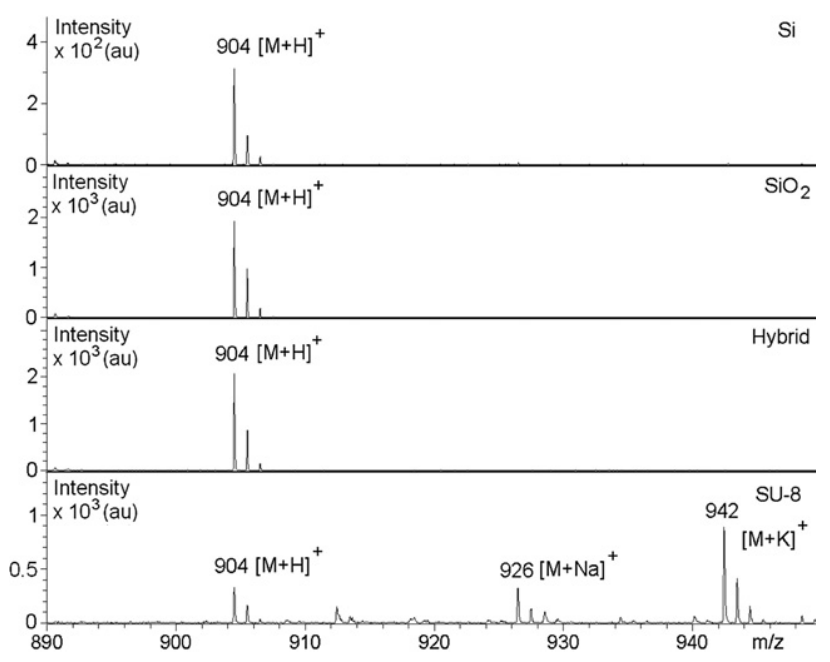

Figure 1. SALDI mass spectra of des-arg ${ }^{9}$-bradykinin (2 pmol) on four different substrates coated with amorphous silicon.

any chemical involved in the process involves sodium or potassium, we think that the most likely explanation is somehow suboptimal composition of PECVD deposited $\alpha$-Si on top of SU-8. The relatively low signal to noise ratios obtained from the $\alpha$-Si coated silicon substrate indicate that our PECVD grown amorphous silicon was not as efficient SALDI active coating as the sputtered $\alpha$-Si used by Alimpiev et al. [16], who reported it to be similar to porous silicon in ionization efficiency when grown on top of a silicon wafer. Also, unlike the previous study, which found no difference between a $500 \mathrm{~nm}$ coating of sputtered $\alpha$-Si on either silicon or stainless steel substrates, we find a clear dependency between ionization efficiency and the material of the underlying substrate, with $\alpha$-Si grown on top of silicon producing clearly weaker signals compared with either $\mathrm{SiO}_{2}$ or the hybrid.

Differences between the substrates can be analyzed in terms of thermal properties of the two layer system consisting of the amorphous silicon coating layer and the substrate. The length scale for the absorption of the laser light in the $\alpha$-Si layer can be approximated as 1/a, where $a$ is the absorption coefficient. For $355 \mathrm{~nm}$ wavelength, the absorption coefficient of amorphous silicon is of the order $1 \times 10^{6} \mathrm{~cm}^{-1}$ [27], so the length scale for absorption is in the order $10 \mathrm{~nm}$, which is several times smaller than the thickness of the coating. The effect of the thin layer of native oxide that forms on the surface of the coating layer has been excluded from this order of magnitude analysis.

The length scale for thermal diffusion during the laser pulse can be approximated as:

$$
d_{\text {diffusion }} \approx \sqrt{\left(k t / c_{p} \rho\right)},
$$

where $\mathrm{k}$ is the thermal conductivity, $\mathrm{t}$ the pulse duration, $\rho$ is the density, and $c_{p}$ is the specific heat capacity.
Using room-temperature values for crystalline silicon, $\left(\mathrm{k}=148 \mathrm{~W} \mathrm{~m}^{-1} \mathrm{~K}^{-1}, \mathrm{c}_{\mathrm{p}}=714 \mathrm{~J} \mathrm{~kg}^{-1} \mathrm{~K}^{-1}, \rho=2330 \mathrm{~kg}\right.$ $\left.\mathrm{m}^{-3}\right)$, and silicon dioxide $\left(\mathrm{k}=1.4 \mathrm{~W} \mathrm{~m}^{-1} \mathrm{~K}^{-1}, \mathrm{c}_{\mathrm{p}}=746\right.$ $\mathrm{J} \mathrm{kg}^{-1} \mathrm{~K}^{-1}, \rho=2200 \mathrm{~kg} \mathrm{~m}^{-3}$ ) [28], the thermal diffusion length during $7 \mathrm{~ns}$ laser pulse can be approximated to be $\approx 800 \mathrm{~nm}$ for silicon and $\approx 80 \mathrm{~nm}$ for silicon dioxide. Most of the laser energy is then absorbed into the thin $\alpha$-Si coating layer, but during the pulse, the heat diffuses also to the substrate. On the silicon substrate, much of the heat is lost into the substrate because of the high thermal conductivity of silicon, while on the hybrid and $\mathrm{SiO}_{2}$ substrates, which both have much lower heat conductivity than silicon, the thermal insulation allows the surfaces to reach higher temperatures for longer periods of time. A similar effect has also been reported by Schürenberg et al. [29], who discussed the superior performance of $35 \mathrm{~nm}$ TiN nanoparticles compared with $1 \mu \mathrm{m}$ TiN microparticles.

The $\mathrm{SiO}_{2}$ and the hybrid substrates were quite similar in performance, with the hybrid substrate producing slightly stronger signals, and also required less laser power. AFM analysis revealed that the $\alpha$-Si coating layer was slightly rougher on top of the hybrid, rms roughness being $0.4 \mathrm{~nm}$ on top of the hybrid compared with $0.1 \mathrm{~nm}$ on top of both silicon and $\mathrm{SiO}_{2}$, which might contribute toward improved ionization by several roughness related ionization mechanisms [17]. Another possible contributing factor is related to surface restructuring and melting [8, 22], which has been claimed to be a key factor in the desorption process in SALDI. We observed that on the $\alpha$-Si coated hybrid surface, clear marks were created upon exposure to the laser even at fluences that were not able to leave any visible marks on the $\mathrm{Si}$ and $\mathrm{SiO}_{2}$ substrates. Scanning electron microscopy of the $\alpha$-Si coated hybrid, shown at Figure 2, reveals that the $\alpha$-Si coating layer had delaminated into micrometer sized domains and gathered in small clusters around the edges, exposing the underlying hybrid material in the process. A possible reason for the greater tendency of morphologic changes for $\alpha$-Si on top of the hybrid material is thermally induced stress caused by the high temperatures during the laser pulse and the great thermal expansion coefficient mismatch between $\alpha$-Si $\left(\approx 2.6 \mathrm{ppm} / \mathrm{K}\right.$ at $\left.20^{\circ} \mathrm{C}\right)$ and the hybrid $\left(\approx 100-130 \mathrm{ppm} / \mathrm{K}\right.$ at $\left.20^{\circ} \mathrm{C}\right)$, while a corresponding mismatch does not exist between $\alpha$-Si and $\mathrm{SiO}_{2}(\approx 0.6$ $\mathrm{ppm} / \mathrm{K}$ at $20^{\circ} \mathrm{C}$ ). Relieving this stress could then facilitate surface restructuring during the laser pulse, which

Table 1. Effect of substrate material on signal intensity of des$\arg ^{9}$-bradykinin ( $\left.2 \mathrm{pmol}\right)$ on three different substrates coated with $40 \mathrm{~nm}$ layer of amorphous silicon. The \pm values describe 10 measurements on different sample deposition spots on the same sample plate

\begin{tabular}{lcrc}
\hline Substrate & Intensity (au) & \multicolumn{1}{c}{$\mathrm{S} / \mathrm{N}$} & Roughness rms (nm) \\
\hline \hline $\mathrm{Si}$ & $300 \pm 50$ & $68 \pm 10$ & 0.1 \\
$\mathrm{SiO} 2$ & $1500 \pm 190$ & $310 \pm 41$ & 0.1 \\
Hybrid & $2000 \pm 70$ & $440 \pm 15$ & 0.4 \\
\hline
\end{tabular}




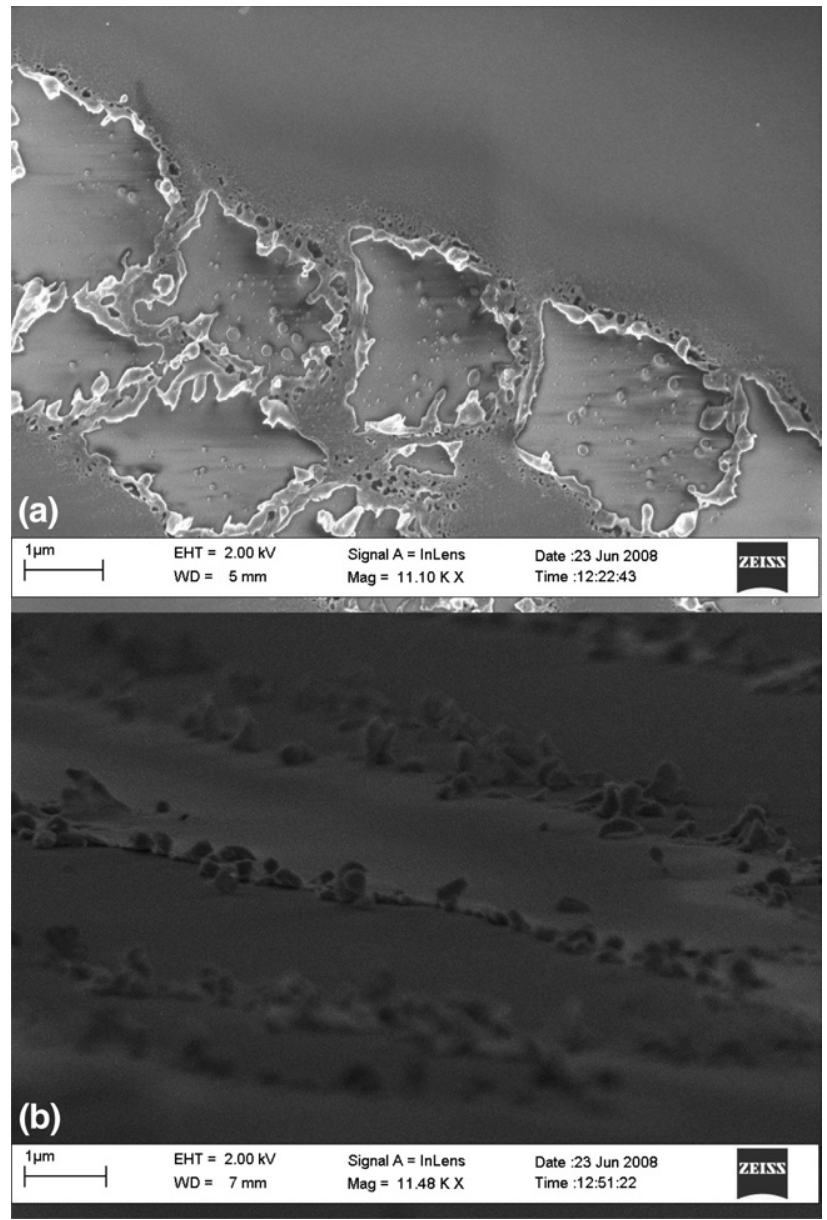

Figure 2. SEM images of $\alpha$-Si layer on top of hybrid after exposure to laser (a) top view (b) side view. No marks were left on $\alpha$-Si layer on top of silicon or silicon dioxide substrates with this laser power.

in turn could contribute toward desorption of the analytes. However, the appearance of this type of large scale structural change was not a necessary requirement for successful desorption-ionization.

The inorganic-organic hybrid material was chosen as the substrate for all subsequent experiments because it gave strong analyte signals and is also easily malleable, unlike the second best substrate material silicon dioxide.

Substrate topography. The effect of surface topography was studied by applying the same amorphous silicon coating on planar hybrid, hybrid micropillars $(\approx 2 \mu \mathrm{m})$ and hybrid nanopillars $(\approx 500 \mathrm{~nm})$. We also utilize the properties of the inorganic-organic hybrid material by combining these basic topographies with nanopores that are known to form when such materials are exposed to oxygen plasma [30]. We are currently still in the process of studying the nature of these pores, but the pore sizes are thought to range from few nanometers to few tens of nanometers. It is worth emphasizing that since the $\alpha$-Si coating comes on top of these pores, we view them more as a modification of the substrate and not as something that is directly comparable to the pores of, for example, porous silicon. The analyte was 10 pmol of des-arg ${ }^{9}$-bradykinin, and the same laser power, optimized for the planar and nonporous topography, was used for all surfaces. The results of this experiment, obtained from three separate measurements on each sample plate, are shown in Table 2. The $\alpha$-Si coating thickness in the experiment summarized in Table 2 was $80 \mathrm{~nm}$, but $8 \mathrm{~nm}$ and $40 \mathrm{~nm}$ coatings were also tried. The surfaces with a $40 \mathrm{~nm}$ coating gave very similar signals compared with the surfaces with $80 \mathrm{~nm}$ of $\alpha$-Si, while the surfaces with only an $8 \mathrm{~nm}$ coating gave somewhat weaker signals by a factor of about two, probably because of insufficient absorption of the laser light.

The nanopillar substrate gave clearly superior signal intensities compared with the planar substrate, while the micropillars were actually detrimental to obtaining strong signals. This result is in agreement with previous publications reporting submicrometer structures and roughness to be conducive for efficient ionization [14, 17]. The reason for decreased signal intensities on the micropillared sample plates could not be determined from the geometries used, but uneven distribution of the analyte during droplet drying (increased on the bottom compared with the tops and sidewalls) combined to the uneven distribution of laser energy (increased on the top parts of the pillars due to shadowing) is a plausible explanation. The RIE oxygen plasma treatment before the $\alpha$-Si deposition resulted in significantly increased signal intensities on all three basic topographies, which made the porous nanopillars, shown in Figure 3, the substrate that gave the strongest analyte signals. The porosity of the substrate contributes by further lowering the thermal conductivity, as has been reported for porous silicon [31] and other materials with cavities [32]. The roughness of the RIE treated sample plates also increased, and AFM analysis performed on the planar and RIE treated hybrid with 40 nm $\alpha$-Si coating showed that the rms roughness had increased to $8.7 \mathrm{~nm}$, the dominant features being aggregates typically about $50 \mathrm{~nm}$ in lateral dimensions and 20 $\mathrm{nm}$ in height, visibly similar to those reported for other polymeric materials [33]. Finally, growing a coating layer on top of a porous substrate also dramatically increases the coating-substrate interfacial area, leading

Table 2. Effect of substrate topography on signal intensity of des-arg ${ }^{9}$-bradykinin (10 pmol) on hybrid sample plates coated with $80 \mathrm{~nm}$ layer of $\alpha$-Si. The \pm values describe three measurements on different sample deposition spots on the same sample plate

\begin{tabular}{lcc}
\hline Hybrid topography & $\begin{array}{c}\text { Untreated } \\
\text { intensity (au) }\end{array}$ & $\begin{array}{c}\text { RIE treated } \\
\text { intensity (au) }\end{array}$ \\
\hline \hline Planar & $7300 \pm 500$ & $44000 \pm 5000$ \\
Micropillars & $2600 \pm 300$ & $5000 \pm 200$ \\
Nanopillars & $22800 \pm 600$ & $65300 \pm 7400$ \\
\hline
\end{tabular}




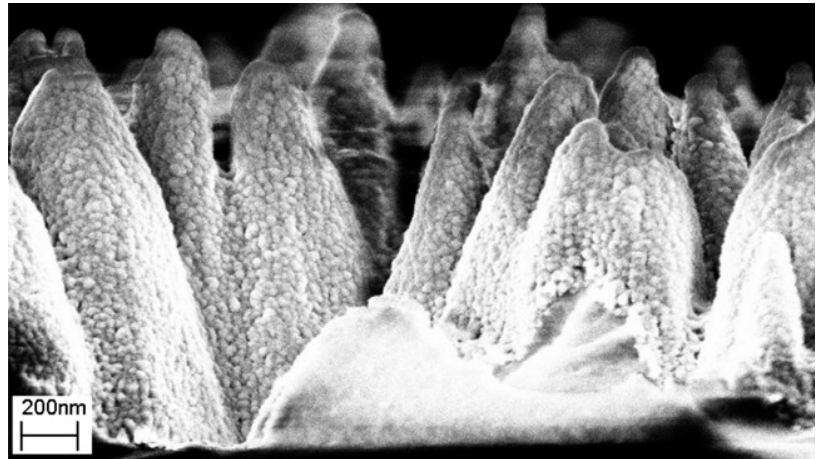

Figure 3. RIE treated hybrid nanopillars coated with $80 \mathrm{~nm}$ $\alpha$-Si.

to a corresponding enhancement of the interfacial energy, which could enhance the tendency for morphologic transitions if the interfacial interaction becomes unfavorable because of the thermal stress during the laser pulse.

Coating material. The effect of the material properties of the coating layer was investigated by applying different thin film coatings on top of the same substrate (nonporous hybrid nanopillars). Nonporous substrates were chosen because of the substantially different way ALD and PECVD deposited thin films conform on porous substrates. The coatings that were tested were ALD titania and alumina, and PECVD $\alpha$-Si and silicon nitride. The nominal thickness of each coating was between $40 \mathrm{~nm}$ and $60 \mathrm{~nm}$. The analyte was $50 \mathrm{pmol}$ of des-arg'-bradykinin and citric acid additive $(1 \mathrm{nmol})$ as an additional proton source was also tested in conjunction with the different coatings.

On uncoated, alumina coated, silicon nitride coated, or uncoated hybrid nanopillars, either no signal or a very weak trace signal was detected with or without the citric acid additive. On the titania coated surface, a weak signal consisting of potassium adducts as well as protonated analytes was obtained without the citric acid additive. However, with the citric acid additive, a strong molecular ion peak was detected instead (Figure S2, Supplementary Material), consistent with the results of Chen et al. [7], who also studied LDI-MS on titania utilizing an additional proton source.

As a summary, $\alpha$-Si was unique among the coating materials that were tested in its ability to produce good quality analyte signals without any additives while titania coating allowed good signals with a citric acid additive, and the other coatings only gave weak trace signals. We suggest that the results can be understood as the combination of the surface chemistry and UV-absorptivity of the coating layer, so that only $\alpha$-Si combines favorable surface chemistry to sufficient UV-absorptivity, while titania has sufficient UV-absorptivity but inefficient surface chemistry for producing protonated or otherwise ionized species without additives, and the other surfaces (alumina, silicon nitride, native hybrid surface) lack at least the necessary
UV-absorptivity, since no signal was obtained from them even with the additional proton source. Indeed, $\alpha-\mathrm{Si}$ surfaces have been shown [16] to have both the surface chemistry and the electrical properties necessary for transferring protons from the surface to the analyte.

\section{SALDI-MS}

RIE treated planar hybrid and RIE treated hybrid nanopillars coated with $\alpha$-Si, were the two most promising sample plates of this study, and they were tested in SALDI-MS of peptides, drug molecules, and small proteins. Mass spectra of a mixture of five different peptides (des-arg'-bradykinin, angiotensin I, angiotensin II, bombesin, and substance-P, 5 pmol each), obtained using $\alpha$-Si coated RIE-porous planar hybrid and MALDI are shown in Figure 4. Overall, the spectra are similar, with the greatest differences being somewhat weaker signal intensities and greatly reduced background peaks in the SALDI spectrum. The detection limits were evaluated for des-arg ${ }^{9}$-bradykinin and were in the high attomole range for $\alpha$-Si coated RIE-porous hybrid nanopillars (Figure S3, Supplementary Material). The corresponding detection limit for $\alpha$-Si coated RIE-porous planar hybrid was in the few femtomole range.

The suitability of our sample plates for analysis of drug molecules was tested with verapamil, propranolol, and midazolam. Figure 5 shows the mass spectra of a mixture containing 1 pmol of verapamil and 5 pmol each of propranolol and midazolam obtained by SALDI using $\alpha$-Si coated RIE-porous hybrid nanopillars sample plates and MALDI. The SALDI spectrum was clear of interfering background peaks and only contained five identifiable peaks: Propranolol at $\mathrm{m} / \mathrm{z}=260$, midazolam at $m / z=326$, verapamil at $m / z=455$, and two known verapamil fragments at $m / z 165$ and 303 . The partial fragmentation of verapamil analytes could not be avoided on these sample plates at laser fluences necessary for efficient ionization, but the fragmentation remained at a modest level, the most abundant ions detected being the protonated intact molecular ions. The MALDI spectrum also contains all the analyte peaks, but the many matrix ion peaks make the spec-

(a)

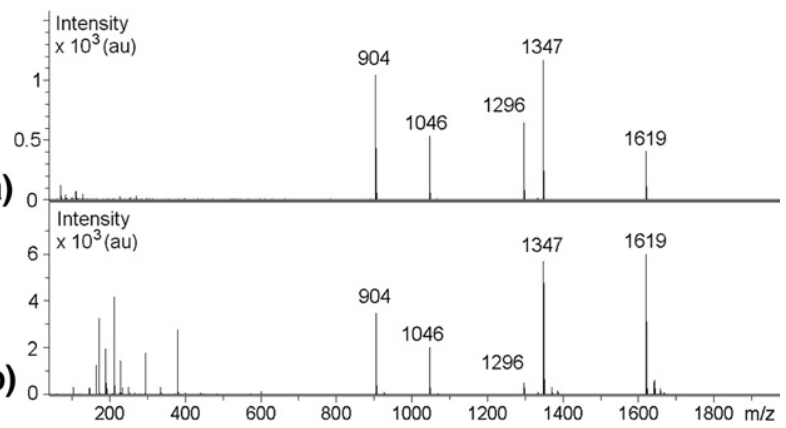

Figure 4. Peptide mass spectra obtained by (a) SALDI-MS, and (b) MALDI-MS. 
trum unnecessarily complex compared with the SALDI spectrum.

Figure 6 shows the mass spectrum of $50 \mathrm{pmol}$ of 12 kDa cytochrome $c$ obtained on $\alpha$-Si coated RIE treated planar hybrid. SALDI-MS of small proteins was possible on our sample plates with a citric acid additive, but not on proteins dissolved in pure water. The largest protein we have successfully detected so far using SALDI-MS on this sample plate is $\beta$-lactoglobulin with mass $18.4 \mathrm{kDa}$.

\section{Sample Plate Aging}

Alimpiev et al. [16] reported that in their experiments, SALDI activity of $\alpha$-Si decayed after few days of exposure to the ambient air and could only be regenerated by laser shots at very high fluences. However, we did not observe such an effect with the PECVD grown $\alpha$-Si used in this study, and the performance of the sample plates did not deteriorate greatly during exposure to ambient air. Good quality signals were obtained from des-arg ${ }^{9}$-bradykinin on 1 day-, 1 month-, 3 month-, and 8-month-old sample plates with both $10 \times 50$ laser shot spectra and single laser shot spectra. The main effect we noticed that aging had on our sample plates was decreasing contact angle over time, probably because of surface oxidation, leading to the analyte being deposited over a larger area. The signal intensities on the oldest 8-month-old sample plates were about half of those on freshly prepared sample plates (Figure S4, Supplementary Material).

\section{Conclusions}

Matrix-free SALDI-MS using two layered structures was investigated for analytical applications and as a tool for experimental studies of surface-assisted laser desorption ionization. Substrates with low thermal conductivity were superior to higher thermal conductivity substrates. For the coating layer, the combination of UV-absorptivity and favorable surface chemistry made amorphous silicon superior to other coating that were

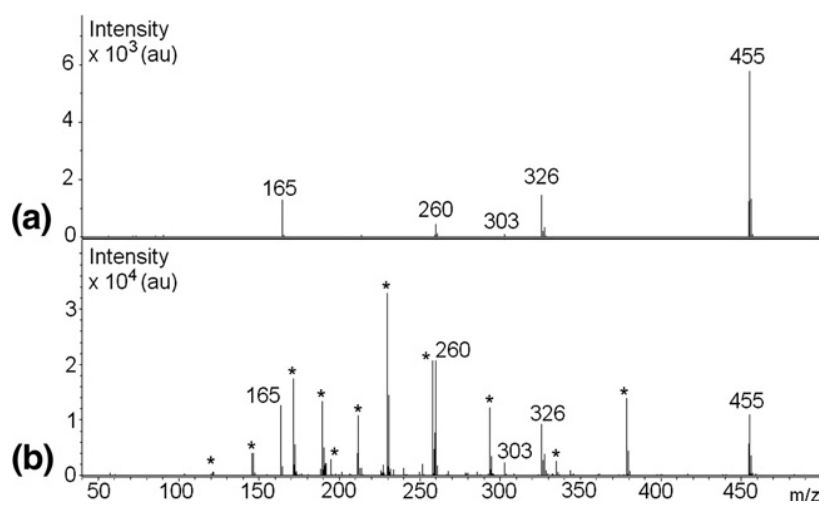

Figure 5. Drug mass spectra obtained by (a) SALDI-MS, and (b) MALDI-MS. Prominent matrix peaks are marked with an asterisk.

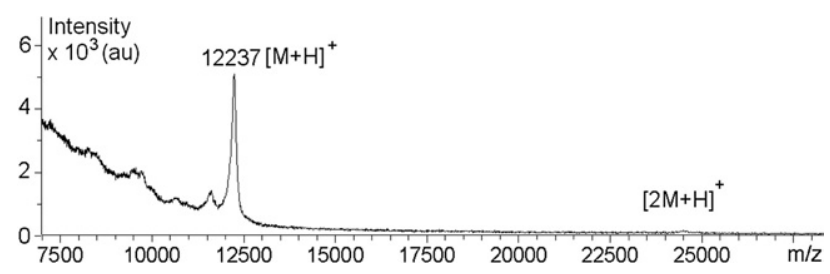

Figure 6. SALDI-MS spectrum of $50 \mathrm{pmol}$ of cytochrome $c$ with $1 \mathrm{nmol}$ of citric acid additive.

tested. The inorganic-organic hybrid was found to be a promising material for SALDI sample plates, and amorphous silicon coated RIE treated planar hybrid and hybrid nanopillars gave the strongest SALDI-MS signals of the sample plates tested in this study. With the current design, we demonstrated a combination of high sensitivity peptide analysis, background-free drug molecule analysis, and the analysis of small proteins, which compares well overall with other published SALDI sample plates. The sample plates are also fully compatible with fabrication processes for microfluidic channels; we are currently working towards combining microfluidic functionalities with SALDI-MS.

\section{Acknowledgments}

The authors thank Dr. K. Grigoras for help with ALD and Mr. N. Chekurov for help with AFM measurements. V.J. and L.S. received financial support from the Finnish National Graduate School in Nanoscience (NGS-NANO); S.A. received financial support from the Graduate School in Electrical and Communications Engineering, and L.L. from the Graduate School of Chemical Sensors and Microanalytical Systems (CHEMSEM). The authors acknowledge partial funding for this work by Biofunctional Microchips (no. 40,380/06) project of the Finnish Funding Agency for Technology and Innovation (TEKES) and The Academy of Finland.

\section{Appendix A Supplementary Material}

Supplementary material associated with this article may be found in the online version at doi:10.1016/ j.jasms.2009.05.013.

\section{References}

1. Wei, J.; Buriak, J. M.; Siuzdak, G. Desorption-Ionization Mass Spectrometry on Porous Silicon. Nature 1999, 399, 243-246.

2. Karas, M.; Hillenkamp, F. Laser Desorption Ionization of Proteins with Molecular Masses Exceeding 10,000 Daltons. Anal. Chem. 1988, 60, 2301-2303.

3. Shen, Z.; Thomas, J. J.; Averbuj, C.; Broo, K. M.; Engelhard, M.; Crowell, J. E.; Finn, M. G.; Siuzdak, G. Porous Silicon as a Versatile Platform for Laser Desorption/Ionization Mass Spectrometry. Anal. Chem. 2001, 73, 612-619.

4. Trauger, S. A.; Go, E. P.; Shen, Z.; Apon, J. V.; Compton, B. J.; Bouvier, E. S. P.; Finn, M. G.; Siuzdak, G. High Sensitivity and Analyte Capture with Desorption/Ionization Mass Spectrometry on Silylated Porous Silicon. Anal. Chem. 2004, 76, 4484-4489.

5. Thomas, J. J.; Shen, Z.; Crowell, J. E.; Finn, M. G.; Siuzdak, G. Desorption/Ionization on Silicon (DIOS): A Diverse Mass Spectrometry Platform for Protein Characterization. Proc. Nat. Acad. Sci. U.S.A. 2001 98, 4932-4937.

6. Seino, T.; Sato, H.; Yamamoto, A.; Nemoto, A.; Torimura, M.; Tao, H. Matrix-Free Laser Desorption/Ionization-Mass Spectrometry Using Self-Assembled Germanium Nanodots. Anal. Chem. 2007, 79, 4827-4832. 
7. Chen, C.-T.; Chen, Y.-C. Desorption/Ionization Mass Spectrometry on Nanocrystalline Titania Sol-Gel-Deposited Films. Rapid Commun. Mass Spectrom. 2004, 18, 1956-1964.

8. Wada, Y.; Yanagishita, T.; Masuda, H. Ordered Porous Alumina Geometries and Surface Metals for Surface-Assisted Laser Desorption/Ionization of Biomolecules: Possible Mechanistic Implication of Metal Surface Melting. Anal. Chem. 2007, 79, 9122-9127.

9. Sainiemi, L.; Keskinen, H.; Aromaa, M.; Luosujärvi, L.; Grigoras, K.; Kotiaho, T.; Mäkelä, J. M.; Franssila, S. Rapid Fabrication of High Aspect Ratio Silicon Nanopillars for Vhemical Analysis. Nanotechnology 2007, 18, 505303.

10. Tanaka, K.; Waki, H.; Idao, Y.; Akita, S.; Yoshida, Y.; Yoshida, T. Protein and Polymer Analyses up to $\mathrm{m} / \mathrm{z} 100,000$ by Laser Ionization Time-ofFlight Mass Spectrometry. Rapid Commun. Mass Spectrom. 1988, 2, 151-153.

11. Sunner, J.; Dratz, E.; Chen, Y.-C. Graphite Surface-Assisted Laser Desorption/Ionization Time-of-Flight Mass Spectrometry of Peptides and Proteins from Liquid Solutions. Anal. Chem. 1995, 67, 4335-4342.

12. Go, E. P.; Apon, J. V.; Luo, G.; Saghatelian, A.; Daniels, R. H.; Sahi, V.; Dubrow, R.; Cravatt, B. F.; Vertes, A.; Siuzdak, G. Desorption/Ionization on Silicon Nanowires. Anal. Chem. 2005, 77, 1641-1646.

13. Finkel, N. H.; Prevo, B. G.; Velev, O. D.; He, L. Ordered Silicon Nanocavity Arrays in Surface-Assisted Desorption/Ionization Mass Spectrometry. Anal. Chem. 2005, 77, 1088-1095.

14. Okuno, S.; Arakawa, R.; Okamoto, K.; Matsui, Y.; Seki, S.; Kozawa, T.; Tagawa, S.; Wada, Y. Requirements for Laser-Induced Desorption/ Ionization on Submicrometer Structures. Anal. Chem. 2005, 77, 53645369.

15. Cuiffi, J. D.; Hayes, D. J.; Fonash, S. J.; Brown, K. N.; Jones, A. D. Desorption-Ionization Mass Spectrometry Using Deposited Nanostructured Silicon Films. Anal. Chem. 2001, 73, 1292-1295.

16. Alimpiev, S.; Grechnikov, A.; Sunner, J.; Karavanskii, V.; Simanovsky, Ya.; Zhabin, S.; Nikiforov, S. On the Role of Defects and Surface Chemistry for Surface-Assisted Laser Desorption Ionization from Silicon. J. Chem. Phys. 2008, 128, 014711.

17. Alimpiev, S.; Nikiforov, S.; Karavanskii, V.; Minton, T.; Sunner, J. On the Mechanism of Laser-Induced Desorption-Ionization of Organic Compounds from Etched Silicon and Carbon Surfaces. J. Chem. Phys. 2001, 115, 1891-1901.

18. Lo, C.-Y.; Lin, J-Y.; Chen, W.-Y; Chen, C.-T; Chen, Y.-C. Surface Assisted Laser Desorption/Ionization Mass spectrometry on Titania Nanotube Arrays. J. Am. Soc. Mass Spectrom. 2008, 19, 1014-1020.
19. Shin, S. J.; Choi, D.-W.; Kwak, H.-S.; Lim, G. I.; Choi, Y. S. Matrix-Free Laser Desorption/Ionization on Vertically Aligned Carbon Nanotube Arrays. Bull. Korean Chem. Soc. 2006, 27, 581-583.

20. Kruse, R. A.; Li, X.; Bohn, P. W.; Sweedler, J. V. Experimental Factors Controlling Analyte Ion Generation in Laser Desorption/Ionization Mass Spectrometry on Porous Silicon. Anal. Chem. 2001, 73, 3639-3645.

21. Luo, G.; Chen, Y.; Siuzdak, G.; Vertes, A. Surface Modification and Laser Pulse Length Effects on Internal Energy Transfer in DIOS. J. Phys. Chem. B 2005, 9, 24450-24456.

22. Northen, T. R.; Woo, H.-K.; Northen, M. T.; Nordström, A.; Uritboonthail, W.; Turner, K. L.; Siuzdak, G. High Surface Area of Porous Silicon Drives Desorption of Intact Molecules. J. Am. Soc. Mass Spectrom. 2007, 18, 1945-1949.

23. Liu, Q.; He, L. Quantitative Study of Solvent and Surface Effects on Analyte Ionization in Desorption Ionization on Silicon (DIOS) Mass Spectrometry. J. Am. Soc. Mass Spectrom. 2008, 19, 8-13.

24. Aura, S.; Sikanen, T.; Kotiaho, T.; Franssila, S. Novel Hybrid Material for Microfluidic Devices. Sens. Actuators B 2008, 132, 397-403.

25. Haas, K.-H. Hybrid Inorganic-Organic Polymers Based on Organically Modified Si-Alkoxides. Adv. Eng. Mater. 2000, 9, 571-582.

26. Aura, S.; Jokinen, V.; Sainiemi, L.; Baumann, M.; Franssila, S. UVEmbossed Inorganic-Organic Hybrid Nanopillars for Bioapplications. J. Nanosci. Nanotechnol., in press.

27. Caputo, D.; de Cesare, G.; Irrera, F.; Palma, F. Solar-Blind UV Photodetectors for Large Area Applications. IEEE Trans. Electron. Devices 1996, 43, 1351-1356.

28. Lide, D. R. CRC Handbook of Chemistry and Physics 76th edition; CRC Press, Inc.: Boca Raton, 1995, 12-178.

29. Schürenberg, M.; Dreiseward, K.; Hillenkamp, F. Laser Desorption/ Ionization Mass Spectrometry of Peptides and Proteins with Particle Suspension Matrixes. Anal. Chem. 1999, 71, 221-229.

30. Shamiryan, D.; Baklanov, M. R.; Vanhaelemeersch, S.; Maex, K. Comparative Study of SiOCH Low-k Films with Varied Porosity Interacting with Etching and Cleaning Plasma. J. Vac. Sci. Technol. B 2002, 20, 1923-1928.

31. Gesele, G.; Linsmeier, J.; Drach, V.; Fricke, J.; Arens-Fischer, R. J. Temperature Dependent Thermal Conductivity of Porous Silicon. Phys. D Appl. Phys. 1997, 30, 2911-2916.

32. Hu, X. J.; Prasher, R.; Lofgree, K. Ultralow Thermal Conductivity of Nanoparticle Packed Bed. Appl. Phys. Lett. 2007, 91, 203113.

33. Walther, F.; Davydovskaya, P.; Zürcher, S.; Kaiser, M.; Herberg, H. Gigler, A. M.; Stark, R. W. Stability of the Hydrophilic Behavior of Oxygen Plasma Activated SU-8. J. Micromech. Microeng. 2007, 17, $524-$ 531. 